Review

\title{
Novel contributors to $B$ cell activation during inflammatory CNS demyelination; An oNGOing process
}

\author{
Olympia Damianidou1\#, Paschalis Theotokis#, Nikolaos Grigoriadis ${ }^{1}$, Steven Petratos ${ }^{2}$ \\ 1. B' Department of Neurology, Laboratory of Experimental Neurology and Neuroimmunology, AHEPA University Hospital, Thessaloniki 54636, Macedonia, \\ Greece. \\ 2. Department of Neuroscience, Central Clinical School, Monash University, Prahran, Victoria 3004, Australia.
}

\#These authors contributed equally to this manuscript.

$\triangle$ Corresponding author: Dr Steven Petratos, E-mail: steven.petratos@monash.edu; Tel.: +613 99020191.

() The author(s). This is an open access article distributed under the terms of the Creative Commons Attribution License (https://creativecommons.org/licenses/by/4.0/). See http:/ /ivyspring.com/terms for full terms and conditions.

Received: 2021.08.21; Accepted: 2021.11.11; Published: 2022.01.01

\begin{abstract}
Over the past two decades, the development of targeted immunotherapeutics for relapsing-remitting multiple sclerosis has been successfully orchestrated through the efficacious modulation of neuroinflammatory outcomes demonstrated in the experimental autoimmune encephalomyelitis (EAE) model. In this model, the focus of developing immunomodulatory therapeutics has been demonstrated through their effectiveness in modifying the pro-inflammatory Thl and Th17-dependent neuropathological outcomes of demyelination, oligodendrocytopathy and axonal dystrophy. However, recent successful preclinical and clinical trials have advocated for the significance of $B$ cell-dependent immunopathogenic responses and has led to the development of novel biologicals that target specific $B$ cell phenotypes. In this context, a new molecule, B-cell activating factor (BAFF), has emerged as a positive regulator of $B$ cell survival and differentiation functioning through various signaling pathways and potentiating the activity of various receptor complexes through pleiotropic means. One possible cognate receptor for BAFF includes the Nogo receptor $(\mathrm{NgR})$ and its homologs, previously established as potent inhibitors of axonal regeneration during central nervous system (CNS) injury and disease. In this review we provide current evidence for BAFF-dependent signaling through the NgR multimeric complex, elucidating their association within the CNS compartment and underlying the importance of these potential pathogenic molecular regulators as possible therapeutic targets to limit relapse rates and potentially MS progression.
\end{abstract}

Key words: B cell-activating factor, Nogo receptor 1, autoimmune demyelination

\section{Introduction}

Multiple Sclerosis (MS) is a chronic inflammatory and demyelinating disease of the central nervous system (CNS) with hallmarks of myelin degeneration and axonopathy [1]. Although its actual cause remains still unknown, various environmental factors such as vitamin D deficiency, smoking, Epstein-Barr virus infection, and genetic factors have been accused as risk factors of the disease [1].

MS is a heterogeneous disease but presentation of characteristic symptoms generally follow stages of neurological deficits (relapses) and these can be reversed during periods of total or partial recovery (remissions) [2,3]. This clinical presentation is seen in relapsing remitting MS (RRMS) that is the most common ( $85 \%$ of individuals diagnosed) form of the disease $[1,2]$. Gradually, the majority of patients with RRMS will transition into secondary progressive MS (SPMS) having accumulated neural tissue damage and hence disability [2]. In some individuals progressive disease manifests in the initial phases of MS without remissions (primary progressive MS; PPMS) resulting in permanent neurodegeneration and in the rare case of progressive relapsing MS (PRMS) the initial progressive phase is followed by acute relapses with intervals of continuous progression [1, 2]. Finally, the inflammatory and demyelinating findings in clinically isolated syndrome (CIS) and the evidence in radiologically 
isolated syndrome (RIS) are supportive of MS in undiagnosed individuals $[2,3]$.

Numerous clinical trials conducted in participants living with relapsing MS and PPMS have contributed to the understanding of MS pathogenesis allowing for development and management of this heterogeneous disease through targeted disease modifying therapies (DMTs) as the stochastic nature of symptoms evolve [4]. Such trials have contributed to the transition from the original dogma that $\mathrm{T}$ cell-dependent mechanisms should be the target of therapeutics, into a more dynamic model of complex $\mathrm{B}$ and $\mathrm{T}$ cell interactions, contributing to the current $\mathrm{B}$ cell depleting trials which have demonstrated successful clinical treatment through targeted B cell therapies in RRMS [1, 4-6].

\section{B cell dependent mechanisms in Multiple Sclerosis}

Although MS has been considered as an autoreactive $\mathrm{T}$ cell mediated disease for a long period of time, accumulating evidence support the implication of $B$ cells and antibodies in MS pathogenesis $[7,8]$. The oligoclonal bands that are detected after analyzing the cerebrospinal fluid (CSF) of MS patients, the presence of $B$ and plasma cells in MS plaques and the accumulated antibodies and complement components in MS lesions emphasize the important role of B cells in MS pathogenetic mechanisms [8].

As principal mediators of the pro-inflammatory response that leads to the evolution of MS lesions were considered to be promulgated by the CD4+ T cells which produce IFN- $\gamma$ [9]. However, there are also other pathogenic cell subsets which are under the control of Th17 cells, that produce proinflammatory IL-17, IL- 6 and are regulated by IL-23 $[10,11]$. These can effectively recruit more $\mathrm{T}(\mathrm{CD} 4+, \mathrm{CD} 8+, \gamma \delta+), \mathrm{B}$, dendritic, natural killer (NK) cells and activate microglia in the perivascular space [9]. Additionally, further evidence has revealed a loss of function and a decrease of induced FOXP3 expressing CD4+ CD25+ regulatory $\mathrm{T}$ cells (iTregs) subsets in patients with MS indicating their reduced suppressive potential towards the pathological inflammatory responses taking place $[9,12]$.

It is well established that the clinical biomarker detected in up to approximately $80 \%$ of patients has been found to have a CSF enrichment of with fragments of immunoglobulin G1 supporting the involvement of B cells in MS pathogenesis. Apart from the production of immunoglobulins, B cells can contribute to the pathology through cell interactions, their role as antigen presenting cells (APCs), that also present CNS antigens, proinflammatory cytokines
(IFN- $\gamma, \mathrm{TNFa}, \mathrm{IL}-6)$ producers $[9,13]$ or immune response modulators, that can restrict $\mathrm{T}$ cell functions through IL-10 directly or indirectly [14].

In patients with MS, B lymphoid follicles, $\mathrm{T}$ cells and APCs were identified in the meninges and complement was detected in MS lesions, underlying once again their role in the disease pathogenesis and particularly in the progression $[9,15]$. Concerning regulatory B cells (Bregs), their suppressive functions toward inflammation and immune responses by cytokine (IL-10, TGF- $\beta$, IL-35) secretion have not been clarified yet in particular in MS as they have been reported to be decreased, increased or even stable [13]. However, elevated concentrations of the new CD19+ CD25+ type of Breg cells were observed in MS cases in direct contradiction to healthy controls and in relapsing phases compared to MS remissions [13].

It has been shown that the levels of cortical demyelination, meningeal inflammation and the consequent disability are all in direct correlation to $\mathrm{B}$ cell follicle-like structures (FLS) in the meninges of patients with progressive MS [14] that have been found near parenchymal infiltrates and underlying grey matter lesions [16]. A study of patients with SPMS revealed the distribution of FLS in the forebrain, in the deep sulci of the frontal, temporal, insula and cingulate cortex as well as demyelinated lesions of subpial grey matter at some distance away from these foci [17]. According to the "germinal center reaction" hypothesis naïve B cells recruited in the CNS (CXCL13 signaling) may present CNS antigens to Th cells. $\mathrm{B}$ and $\mathrm{T}$ cells then participate (as centroblast and follicular Th, that produce IL-21) in the formation of germinal centers where B to plasma cell maturation takes place and survival factors, that include B cell-activating factor (BAFF) are actively produced [14]. FLS exist either as tertiary/ectopic lymphoid follicles in the case of chronically inflamed organs in autoimmune diseases such as MS, systemic lupus erythematosus (SLE) and rheumatoid arthritis (RA) or as embryological secondary follicles. In the FLS microenvironment of the brain, B cell interactions can influence/sustain humoral and cell immunity intensifying the already existent demyelination, oligodendrocyte loss and brain cortex damage [15] with the help of many activated microglial cells in the outer cortical layers. The inflammatory microenvironment is accompanied by neuronal loss and glial cell damage, mainly in the superficial cortical lamina, close to the outer pial surface [17].

A very important molecule, that acts as a regulator of B cells survival and development, is BAFF (or BlyS), a cytokine that belongs to the TNF family. It is produced by different cell populations, that can include macrophages, monocytes, dendritic, 
other immune cells and of course B as well as T cells $[8,18,19]$. BAFF mediates its functions mainly through direct ligand-specific effects of three different receptors, thereby activating various signaling pathways, but it can also be involved in the development of autoimmunity [20].

Evidence for B cell involvement during neuroinflammation has also emerged from mice following EAE induction. This animal model represents the major hallmarks of the MS disease and pathology primarily through a CD4+ $\mathrm{T}$ cell-mediated autoimmune reaction that follows the MBP, PLP or MOG immunization [9, 21, 22] although CD8+ $\mathrm{T}$ cells constitute the predominant $\mathrm{T}$ cell populations in MS [23]. This is a limitation of the current animal models that are utilized to understand the complexity of MS pathology since CD8+ $\mathrm{T}$ cells (touted as tissue resident memory cells) are observed to be compartmentalized within demyelinating lesions as CD20+ $\mathrm{B}$ cells are and CD4+ $\mathrm{T}$ cells seem to be transient through the course of disease [24]. Indeed, if targeted CD20+ B cells therapeutics are administered in patients with RRMS we can achieve a reduction in CD8+ $T$ cells that are myelin specific [25]. Therefore, there is a need to model the complex immunopathogenesis of MS demyelination. Models that may resemble these patterns of immune cell-associated lesion profiles can be observed in the Theiler's Murine Encephalomyelitis Virus (TMEV) where the chronic progressive disease course is associated with potentiated myelin reactive $\mathrm{CD} 4+$ and $\mathrm{CD} 8+\mathrm{T}$ cells [26] or the adoptive transfer of CD8+ autoreactive $\mathrm{T}$ cells from humanized mice that target PLP to produce profound brain inflammation [27]. The unique interactions however between the innate and compartmentalized adaptive immune cells have not been clearly elucidated and are required to identify highly effective therapeutics that can modulate the demyelinating lesion milieu.

Even though research has been primarily focused on T cell-dependent pathogenesis lately, it is equally important to study the $B$ cell mediated humoral responses in order to better understand the orchestrated immune responses taking place in conditions such as MS. Most versions of EAE lack the involvement of lymphocytes such as B cells in the disease. Nevertheless, administering larger protein antigens or even using B cells in non-immunizationbased models can overcome such limitations [28]. In this context, a study was conducted to reveal how B cells can participate in spontaneous CNS autoimmune disease of 2D2 IgH MOG mice, where spontaneous EAE can be a monophasic or chronic disease, correlated with $\mathrm{T}$ and $\mathrm{B}$ cell clusters formed within meninges and thereby inflammation in the spinal cord
[28]. Other than that, it was shown that in the EAE animal model without CX3CR1, a receptor for the NK cell recruitment with in the CNS, had elevated levels of CD4+ T cells and thus a more severe form of EAE. These findings suggest a possible ameliorating function of CX3CR1 and NK cells in the disease, regulating the levels of CD4+ $\mathrm{T}$ and Th17 cells [9].

\section{The regulation of BAFF during MS and other autoimmune diseases}

High concentrations of BAFF have been observed in patients with various autoimmune diseases, such as RA, SLE, Sjogren's syndrome, systemic sclerosis, as well as in patients with myelin oligodendrocyte glycoprotein antibody (MOG-Ab) associated demyelination (a separate disease from MS and aquaporin-4 IgG-positive NMO) [8, 29]. In the later case, patients had increased levels of $\mathrm{B}$ cell related cytokines such as BAFF that were associated with elevated Th-17, neutrophil, humoral and lymphoid cytokines (CXCL13, 12, 19), indicating BAFF's role not only in B cell immunity but in other inflammatory mechanisms as well [29]. Although the role of BAFF in MS has not been completely elucidated, its increased levels in CSF during relapses and expression in active MS lesions [30] confirm its pathogenic role in the disease. Moreover, in the humoral response against EBV and Mycobacterium avium (MAP) infection, BAFF levels were found to be inversely associated [29].

Chronic autoimmune diseases, such as SLE, Hashimoto thyroiditis, RA, MS are characterized by inflammatory sites with lymphoid neogenesis, which constitute pathological lesions [31, 32]. These regions seem to be enriched with BAFF expression $[18,31]$. Additionally, BAFF is also increased, up to lymphatic tissue levels, in MS plaques and even more in astrocytes of active MS lesions [30]. Furthermore, high amounts of BAFF protein and mRNA levels can be found in monocytes and increased mRNA levels in T and B cells of MS patients [8, 29,31]. Detecting an association between sCD163 (marker of activated monocyte/ macrophages) and BAFF levels in CSF supports the consensus that infiltrating macrophages (in meninges, choroid plexus or CSF) and activated microglia with in the brain are probably related to constant intrathecal production of BAFF and inflammatory mediators [29]. It is considered that the infiltrating macrophages, microglia and dendritic cells might also be possible sources of BAFF, responsible for the proliferation of CNS invasive autoreactive B cells during inflammation [29].

From a genetic point of view, a correlation has been found between an insertion-deletion variant, derived from TNFSF13B, which encodes BAFF, and 
the higher risk for MS, SLE and possibly RA [33]. The product of this mutation is a short transcript, the function of which is not inhibited resulting in excessive production of BAFF. The increased BAFF concentration is related to a rise in the population of $\mathrm{B}$ cells and antibodies, as well as to lower monocyte numbers and therefore a higher propensity exists for the development of autoimmunity [33].

In mice with relapsing-remitting and chronic relapsing EAE the CNS was characterized by a higher expression of BAFF and CXCL13 genes [7]. Follicular dendritic cells, in secondary lymphoid organs, produce CXCL13 to lead B cells into their follicles [34-36]. It has been found that, during EAE, CXCL13 and BAFF mRNAs are induced intracerebrally and CXCL13-producing cells can be detected in the meninges of these mice suggesting that these tissue sites may be responsible for inducing autoimmune disease [7].

Another study in BAFF-deficient mice revealed that treating the animals with a recombinant Fc-BAFF protein allows splenic B cell zone formation, B cell maturation and physiological antibody response against $\mathrm{T}$-dependent antigen to occur. These functions were limited whenever the treatment was not applied and the mature B cell population was estimated to be significantly lower. Thus, administering recombinant Fc-BAFF can be used to treat BAFF-deficiency [37].

\section{BAFF system: structure, function, signaling pathways and the association with NgR and autoimmunity}

The two putative ligands, BAFF and A proliferation inducting ligand (APRIL), as well as their three cognate receptors (BAFF-R, TACI and BCMA) cooperatively regulate the BAFF cell signaling pathways. BAFF, also known as BlyS, THANK-1, CD257, TNFSF13B, ZTNF4 and TALL-1, was first detected in 1999 by multiple research groups [38-40]. It is a type II transmembrane protein that consists of 285 amino acids and belongs to the tumor necrosis factor (TNF) family of ligands [38, 41]. The protein is encoded in human by the BAFF gene on chromosome 13 (13q34) [38, 42, 43] with exons: 1, 2 and 3-6 coding for the transmembrane domain and its flanking regions, the furin processing site and the TNF homology domain (which binds to receptors), respectively [38]. As a member of the TNF family BAFF's main functions are related to cell death, differentiation and survival [8]. The molecule is of great importance for B cell survival, development, differentiation, immunoglobulin production and $\mathrm{T}$ cell stimulation [29].

BAFF is found initially as a homotrimer on the cell membrane, but if it gets cleaved by furin protease, it is released as a soluble trimeric cytokine [19, 44]. Even though its receptor-binding domain is trimeric, it is not uncommon for 20 BAFF trimers to assemble through a loop region (flap) into a 60-mer [45, 46]. There is also delta-BAFF, a short variant protein, which instead of connecting with BAFF-R, binds to BAFF resulting in inactive heterotrimeric products and therefore negative regulation of BAFF can ensue [47]. Apart from the homotrimers and delta-BAFF, BAFF can form polymers of various compositions with its structural homolog [8], APRIL [38], which are susceptible to BAFF-targeting therapies [46]. APRIL (also known as TRDL-2, TNFSF-13A, TALL-2 [38]) exists mainly as a soluble molecule [8], but it can be localized to plasma membrane surfaces as part of the hybrid molecule TWE-PRIL (also known as TNFSF12TNFSF13), derived from TWEAK (TNF-related weak inducer of apoptosis) and APRIL gene trans-splicing $[47,48]$.

BAFF production has been observed in most myeloid cells and innate immune cells like macrophages, neutrophils, monocytes, IL2-activated natural killer cells and TLR9-activated plasmacytoid dendritic cells [18], $\mathrm{B}$ and $\mathrm{T}$ cells [44, 49] and radiation-resistant stromal cells $[18,48]$. APRIL is mainly produced by neutrophils, macrophages, monocytes, dendritic cells and T-cells [8]. Additional BAFF and/or APRIL sources seem to be nonhematopoietic cells, like epithelial cells and astrocytes with a possible involvement in B cell maintenance at disease specific sites [18]. Furthermore, BAFF can be detected in non-lymphoid cells such as salivary ductal cells in Sjogren's which might be a key pathogenic role in autoimmune epithelitis [50], airway gland epithelial cells where BAFF and APRIL expression may contribute to local migration, class switch recombination and Ig production by B cells found in the airway [51] or osteoclasts (also express APRIL) [52]. Complementary, vascular cell adhesion molecule 1-positive stromal bone marrow cells (VCAM-1 BMCs) that express the molecule seem to be a cell population that enhances developing $B$ and plasma cells in bone marrow [53] and fibroblast-like synoviocytes that are induced by cytokines like IFN- $\gamma$ and TNF-a to express BAFF may be highly capable to protect B cells from apoptosis in inflammatory microenvironments in vivo $[52,54]$.

The main cognate cell-surface receptors are BAFF receptor-(BAFF-R), the transmembrane activator, calcium-modulator and cyclophilin ligand(TACI) and the B-cell maturation antigen-(BCMA) [8, $55,56]$. In humans, all three of them are found on $B$ cells. BAFF-R is involved in B cell survival, maturation and tolerance [20, 42, 47, 57] being 
expressed on all B cells apart from bone marrow plasma cells, as well as on Tregs and activated T cells $[47,52]$. TACI is important for $\mathrm{T}$ cell-independent responses of $\mathrm{B}$ cells to type I and type II antigens, negative regulation of the $\mathrm{B}$ cell class-switch recombination and arrangement [46]. This receptor can be detected on tonsillar and bone marrow plasma cells, activated CD27- non-germinal center cells, CD27+ memory B cells and naive B cells in tonsils and blood [47]. Human intracellular TACI is expressed by macrophages and after being activated it is found on the cell surface [52]. Evidence exists in support of TACI's capability of inducing B cell apoptosis and thus potentially having an important role in preserving immune system homeostasis [58, 59].

BCMA is produced by spleen, bone marrow [47], plasmablasts, plasma cells, tonsillar memory B cells and germinal center B cells [52] promoting survival [46]. It has a significant role, especially in the survival of bone marrow plasma cells and plasmablasts, at later stages $[60,61]$ and its complex with BCMA can overpower BAFF in the last stages of differentiation [42]. APRIL, on the other hand, interacts with TACI, BCMA and surface heparin sulfate proteoglycan (HSPG) leading to its multimerization and subsequent downstream signaling [44].

BAFF and BAFF-R pathways target canonical or non-canonical NF-kB dependent pathways. The canonical NEMO-dependent pathway requires the IKK complex, activators such as TRAF2, 5 and 6 and yields NF-kB dimers with a p50 subunit $[42,57,62$, 63]. Stimulation of the non-canonical NF-kB2 pathway by BAFF is slow, complex [64] and it depends on the NF- $\mathrm{KB}$ inducing kinase NIK (MAP3K14) activation [20, 65]. If BAFF is not present, NIK binds TRAF3, promoting the proteasomal degradation of NIK by a complex of the cellular inhibitors of apoptosis cIAP1 and 2, TRAF2 and 3. Conversely, when BAFF binds to BAFF-R, the receptors cluster and TRAF3 binds to their intracellular sequence allowing the NIK-TRAF2/3-cIAP1/2 complex disassembly and the TRAF3 proteasomal breakdown [20]. The accumulated NIK molecules phosphorylate IKK1, which phosphorylates NF-kB2 p100, the new target of a ubiquitin ligase, that is cleaved into active p52. P52 binds with relB and can regulate nuclear genes, like ICOSL, a costimulatory ligand for ICOS, from activated $\mathrm{T}$ cells, that promotes follicular Th cells development [20].

Another pathway associated with BAFF-R is the phosphoinositide-3-kinase (PI3K) dependent signaling cascade, which shares common components with the BCR pathway [66, 67]. BAFF-R signals can interplay with various molecules (WIP, CD81 and CD19), resulting in cytoskeleton modification.
Downstream of this pathway, the AKT/mTOR axis activates B cell-specific metabolic reactions involved in their survival and function. In mice, BAFF triggers phosphorylation of AKT [68] and subsequently of the factor FOXO, the inhibitor 4EBP1, GSK3 $\beta$ and the small ribosomal subunit protein $\mathrm{S} 6$ indicating BAFF-R significance in protein synthesis. Moreover, MCL-1 stabilization [68], leads to increased mitochondrial function and ATP production [20] (see Figure 1).

BAFF signaling is also involved in the recombination for immunoglobulin class switching. Even though in the case of T-dependent antigens it requires at least two independent signals, from cytokines and also CD40 [42, 69], in human B cells there is no need for additional CD40 signals with BAFF and APRIL being the main ligands [42, 70]. There is, also, evidence about the $\mathrm{B}$ cell co-receptor complex (CD19, CD21 and CD81), which may be enhanced by BAFF, connecting to $\mathrm{C} 3$ complement component. Opsonized antigens are able to cross-link the BCR to the co-receptor, reducing the point of B cell activation and accelerating T-independent antibody production [42]. BAFF and its cognate receptor are also implicated in $\mathrm{T}$ cell physiology inducing the proliferation of suboptimally stimulated $\mathrm{T}$ cells [42]. BCMA, which primary $B$ cells can obtain under collaborative IL-4 and -6 effects, is associated with increased antigen presentation [42]. Regarding TACI, its presence is important for communication to occur between B cells and BAFF-expressing dendritic cells which will eventually allow dendritic cells to prime CD8+ T cells $[42,71]$.

Changes in circulating levels of BAFF, which are genetically regulated and lead to its increase, seem to be strongly correlated with the risk of autoimmunity, as Genome-wide genetic association (GWAS) studies on MS and SLE patients reveal [20]. Moreover, restricted or limited TACI function can also lead to immunodeficiency or autoimmunity accompanied by lymphoproliferation, thereby TACI deficient humans and mice can develop since BAFF levels no longer are properly regulated [20]. Many animal models representing diseases do exhibit high levels of soluble BAFF. In the case of chemically induced autoimmunity by $\mathrm{HgCl}_{2}$ there is an elevated production of BAFF, and TACI-Ig fusion protein is a suggested therapeutic that neutralizes both BAFF and APRIL inhibiting Hg-induced autoantibody or IgE secretion [72]. In collagen-induced arthritis the overproduction of BAFF by dendritic cells and macrophages may play a crucial role in the pathogenesis of the experimental arthritis [73]. Lastly, in SLE models, BAFF and its receptors are involved in the development of the disease and TACI-Ig seems to be a promising treatment, potentially applicable in 
human autoimmune disease [74]. About (20-50\%) of individuals living with autoimmune diseases have been reported to also exhibit elevated levels of BAFF which have been correlated with pathogenic autoantibodies and disease severity. Indeed elevated APRIL and BAFF/APRIL heterotrimers has been observed in sera, targeting patients' organs and thus indicating a strong possibility for excessive BAFF and APRIL to have a pathogenic role in autoimmune sequelae [52].

\section{NgR complex, signaling pathways, interaction with various components}

It has previously been revealed that there is a correlation between BAFF and Nogo receptor (NgR) signaling complex [75], a member of the leucine rich repeat (LRR) superfamily [76], that binds with high affinity and may potentially function as a BAFF receptor $[48,77]$. The $\mathrm{NgR}$ signaling complex consists of $\mathrm{NgR1}$, the co-receptor LINGO-1 and the neurotrophin receptor p75 (p75NTR) or TROY receptor and it mediates its inhibitory effect against axonal regeneration through binding to Nogo 66 with nanomolar affinity [78-80]. This receptor complex binds with myelin associated inhibitory factors (MAIFs), which are myelin-associated glycoprotein (MAG), oligodendrocyte-myelin glycoprotein (OMgp) and Nogo-A and are found on myelin sheath of oligodendrocytes $[77,81,82]$. Nogo- $A$ is one of the three splice variants (Nogo A, B and C), that have a conserved extracellular region of 66 amino acids [80, 83], which is found between two transmembrane helices allowing the molecule to anchor to the oligodendrocyte membrane [81]. This region is responsible for the Nogo related signals that inhibit neurite outgrowth and promote growth cone collapse $[79,81,84]$.

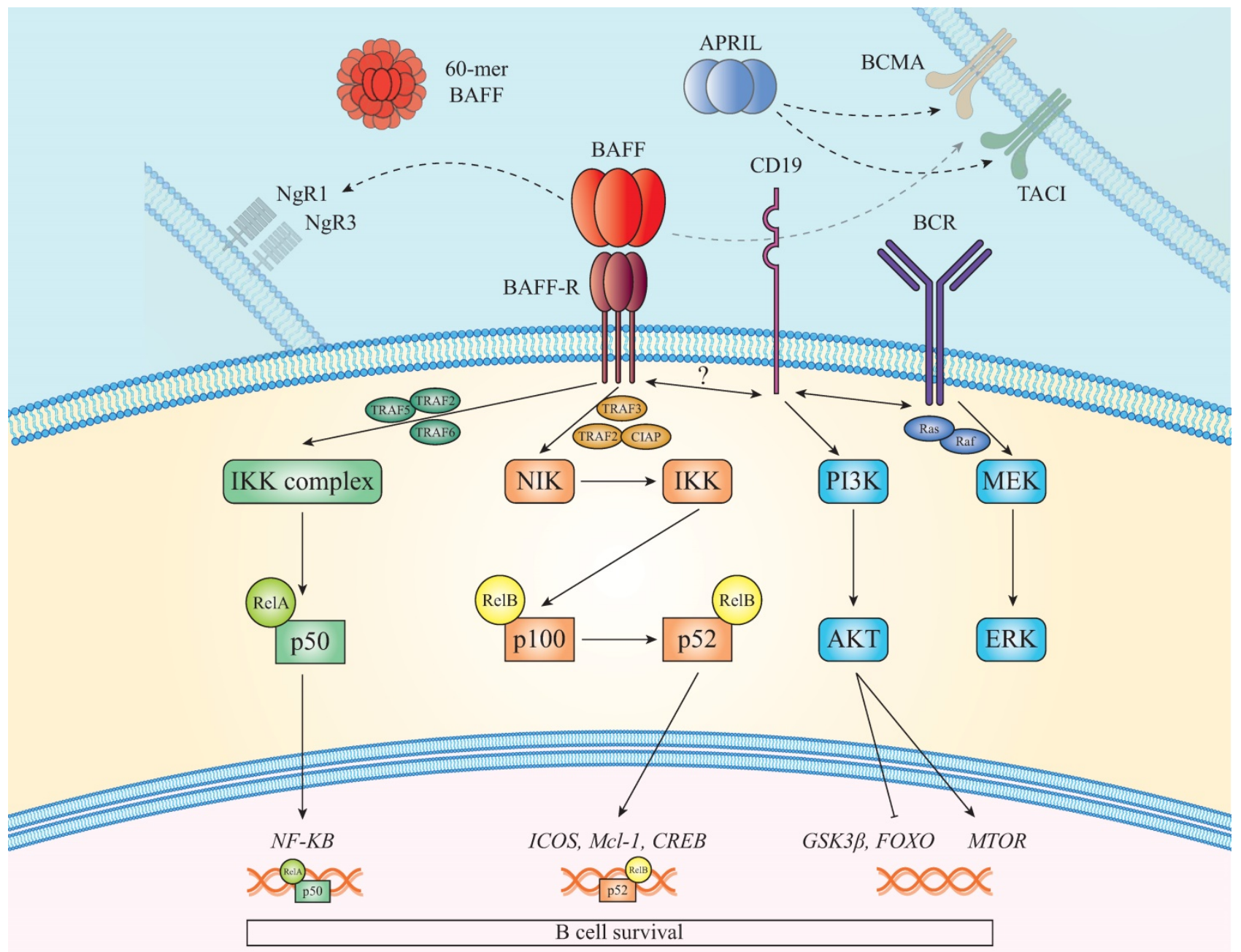

Figure 1. BAFF and BAFF-R signaling pathways. In the presence of BAFF, TRAF3 is intracellularly recruited to BAFF-R allowing NIK to activate the non-canonical NF-KB2 pathway which results in $\mathrm{P} 52 /$ RelB nuclear translocation and activation of cell survival genes. Another pathway activated after BAFF/BAFF-R binding is the canonical that upregulates the expression of various genes, such as NF-KB. Apart from these, PI3K pathway, which shares molecules with the BCR signaling, is additionally activated. Furthermore, it has been shown recently that BAFF interacts with $\mathrm{NgR1}$ and $\mathrm{NgR3}$, promoting $\mathrm{B}$ cell proliferation and maturation. Lastly, TACl and $\mathrm{BCMA}$ can also bind with BAFF and APRIL firing signals that promote antigen presentation and cell survival. 
Apart from neurons, $\mathrm{NgR}$ is also detected on immune cells. More specifically, NgR1 can reduce synaptic plasticity in the cortical gray matter and modulate dendritic spines that are experience dependent [85]. LINGO-1, another LRR superfamily member, is found in CNS neurons and oligodendrocytes inhibiting oligodendrocyte differentiation and myelination [71, 78]. The low affinity neurotrophin receptor p75NTR, is expressed in a plethora of cell types [78]. Its functions comprise cell proliferation, apoptosis, and interestingly the NgR1 and LINGO-1 synergistically inhibit axonal regeneration. Another receptor expressed in neurons is TROY, which substitutes p75NTR within the $\mathrm{NgR}$ complex under certain conditions and is also involved in axon growth suppression [78].

Following the high affinity ligation of the MAIF proteins with $\mathrm{NgR}$, alpha and gamma secretase activity can physiologically modify signaling through intramembranous cleavage of p75NTR (or alternatively TROY [86]) liberating an intracellular signaling peptide, that suppresses axonal growth through activation of Rho GTPase and hence its Rho kinase to limit microfilament and microtubule polymerization [81]. Additionally, gangliosides play a collaborative role in the signaling complex and have also been demonstrated to be important in preventing neuronal growth after spinal growth injury [80, 81]. During neuroinflammation it has been demonstrated that NgR1-dependent signaling within spinal cord and optic nerve axons occurs through RhoA-GTP and the phosphorylation of the Rho-associated coiled-coil containing protein kinase 2 (ROCK II) to alter axonal transport and elicit neurodegeneration through downstream phosphorylation of the collapsin response mediator protein 2 (CRMP2) at the threonine 555 site located at its C-terminus [21, 87]. Moreover, activation of $\mathrm{NgR}$ signaling through the binding of MAIFs present in extracellular myelin debris, can potentiate endogenous inhibitory effects on the maturation of oligodendroglial precursor cells (OPCs) and their recruitment around demyelinated plaques disrupting the remyelination process in progressive MS [77].

Recently, a study revealed that $\mathrm{NgR}$ expression in the CNS of EAE mice may be involved in BAFF signaling pathways which promote B cell proliferation, maturation and differentiation in EAE lesions [77]. The evidence suggests that $\mathrm{NgR1}+$ and $\mathrm{NgR} 3+\mathrm{B}$ cells, mainly found clustered in leptomeningeal infiltrates of lumbosacral spinal cords, can produce antibodies which target spinal cord white matter myelin and the integral myelin membrane glycoproteins [77]. Even though BAFF and $\mathrm{NgR} 1$ binding can result in DRG neurite outgrowth inhibition, it may also lead to the expansion of a subclass of intrathecal NgR1+ and NgR3 + B cells which can propagate neuroinflammatory damage. Thus, it was shown for the first time, that $\mathrm{NgR} 1$ and $\mathrm{NgR} 3$ contribute actively in B cell maturation during EAE and may be involved in BAFF and various other signal transduction events that lead to intrathecal proliferation of these cells. A finding that is also supported by inhibition of $\mathrm{B}$ cell proliferation and differentiation that can result after blocked BAFF binding to $\mathrm{NgR}$ as it was shown that it can be antagonized by the Nogo receptor fusion proteins (NgR-Fc and NgR3-P) [77]. These findings are in agreement with the possible indication of BAFF's involvement in the pathophysiological mechanisms of various autoimmune CNS diseases as increased expression was identified in astrocytes and microglia in patients with MS $[38,75]$.

Moreover, chondroitin sulphate proteoglycans (CSPGs) from reactive astrocytes or T-cells can promote B-cell differentiation and involve in antigen presentation during autoimmunity or even modulate B-cell maturation through binding and activating APRIL. This is in agreement with the fact that NgR1 and $\mathrm{NgR} 3$ can interact with many receptors inducing various signaling pathways for B-cell activation, which is of great significance in MS since its histopathological hallmark indicates the presence of autoreactive B-cells in follicles within the meninges and can lead to progressive neurodegeneration [77]. Furthermore, BAFF seems to be a possible candidate for therapy in MS, since it is produced by astrocytes of the CNS during its pathogenesis and it is associated with cells that have BAFF-R on their surface which is up-regulated in meninges of ectopic lymphoid follicles. Thus, antagonizing BAFF and the following signaling cascade with recombinant $\mathrm{NgR}$ peptides may be one way to inhibit the B-cell proliferative response [77].

\section{Can the therapeutic targeting of BAFF and NgR be developed to limit B cell proliferation/maturation and neurodegeneration in MS?}

The development of the modern MS treatments begins with IFN $\beta$ and glatiramer acetate which were used for the reduction of relapses in relapsing remitting MS (RRMS) cases [88-91], of white matter lesions development and disability accumulation [92, 93]. Monoclonal antibodies such as natalizumab that inhibits lymphocyte invasion in the CNS after blocking adhesion through $\alpha 4 \beta 1$ integrin, lately used oral medications fingolimod, ozanimod and siponimod that are sphingosine-1-phosphate (S1P) 
receptor regulators capable of separating lymphocytes in primary lymphoid organs and teriflunomide, anti-inflammatory dimethyl fumarate, cladribine, mitoxantrone are only some of the recent additions [88-92, 94]. The development of new monoclonal antibodies, like alemtuzumab, as well as B cell depleting therapies with anti-CD20 monoclonal antibody rituximab and ocrelizumab, ofatumumab, anti-CD19 antibody inebilizumab [95] continues enriching the list of currently approved therapies [88-91, 94, 96] (Table 1).

In this context, could the therapeutic targeting of BAFF limit the damaging effect of proliferating B cells in MS? This question seems to concern scientists/ clinicians and it has become a focus of emerging research pathways in B cell depleting MS therapy, since BAFF as well as APRIL potentiate B and plasma cells survival. Experiments with BAFF antagonists have demonstrated favorable outcomes in murine models of RA, Graves' disease and MS, enhancing their appeal as possible treatments for human patients $[52$, 97]. Despite these positive experimental investigations and the clinical data which show that anti-CD20 monoclonal therapeutic antibodies deplete $B$ cells, targeting BAFF and APRIL has important effects on mature plasma cells, since these populations express BCMA but not CD20. Furthermore, rituximab (a well utilized therapeutic agent to reduce CD19+ B cells) can elevate serum BAFF levels, increasing the possibility of immature cells being exposed to high levels of BAFF and leading to autoimmunity. These side effects could be averted by targeting BAFF directly [52].

For this purpose, depleting anti-BAFF monoclonal antibodies and soluble TACI-Fc fusion protein atacicept, which diminish BAFF and APRIL, have been investigated [98]. Early phase clinical trials revealed that atacicept worsened the disease course without a clear etiology. The increased activity in patients indicated that the protective role of $\mathrm{B}$ cells was affected underlying the complexity and the difficulty to target humoral immunity in MS [97-100]. On the other hand, belimumab, a BAFF antibody, has been used in SLE patients with B cell occurring seropositivity [97, 101] and clinical trials have been conducted with tabalumab/ LY2127399 antibody for RRMS, although no indication of treatment effect was confirmed $[97,98]$. In the EAE model, a BAFF - APRIL antagonist (soluble B-cell maturation antigen-Fc) improved the disease course as it was expected [97], whereas mice without BAFF-R had enhanced damage [98].

Although the current MS treatment field seems to be dominated by immunomodulatory therapies, cases of chronic active MS lesions are unresponsive to them underlying an imperative need for new agents, able to modify neurodegenerative lesions [77, 102]. It is worth noting that our recent data derived from the EAE model may partly elucidate the effect of the BAFF protein as a ligand for Nogo receptor (NgR1 and NgR3) expressing B cells. These cells can proliferate and differentiate under the control of BAFF, although provision of the $\mathrm{NgR}$ fusion protein can antagonize this effect [77]. Moreover, such cells, located within leptomeninges, are capable of secreting anti-myelin antibodies whose targets are myelin and oligodendrocytes [77]. The data support that BAFF signaling pathways are involved in neuroinflammation and both $\mathrm{NgR} 1$ and $\mathrm{NgR} 3$ can be of great significance for the neurodegenerative mechanisms that govern the progressive outcomes observed in EAE and MS, suggesting that specifically targeting this interaction on maturing B cells could be a possible target for novel treatment approaches in MS [77].

Table 1. Approved immunomodulatory therapies for MS

\begin{tabular}{llll}
\hline Brand name & Generic name & Target & Function \\
\hline Betaferon (Bayer) & Interferon- $\beta-1 \mathrm{~b}$ & Interferon-a/b receptor & Inflammatory regulation \\
Copaxon (CSL) & Glatimerar acitate & MBP reactive lymphocytes & Prevent myelin specific responses by lymphocytes \\
Rebif (Merck) & Interferon- $\beta-1 \mathrm{a}$ & Interferon-a/b receptor & Inflammatory regulation \\
Avonex (Biogen) & Interferon- $\beta-1 \mathrm{a}$ & Interferon-a/b receptor & Inflammatory regulation \\
Tysabri (Biogen) & Natalizumab & a4-integrin & Reduces lymphocyte trafficking through BBB \\
Gilenya (Novartis) & Fingolimod & Sphingosine-1 phosphatase receptor & Limits lymphocyte trafficking out of lymph nodes \\
Tecfidera (Biogen) & Dimethyl fumarate & Unknown & Protective against oxidative stress \\
Aubagio (Genzyme) & Teriflunomide & Dihydroorotate dehydrogenase & Limits immune cell replication \\
Lemtrada (Genzyme) & Alemtuzumab & CD52+ & T and B lymphocyte depletion \\
Plegridy (Biogen) & Pegylated Interferon- $\beta-1 \mathrm{a}$ (n) & Interferon-a/b receptor & Inflammatory regulation \\
Ocrevus (Roche) & Ocrelizumab & CD20+ lymphocytes & Inflammatory regulation reducing CNS attacks \\
Mavenclad (Merck) & Cladribine tablets & DNA of proliferating immune cells & inflammatory regulation \\
Mayzent (Novartis) & Siponimod & Selective binding of sphingosine-1-phosphatase & Prevents immune cell egress from lymph node \\
& & receptor & \\
\hline
\end{tabular}


Collectively, B cell therapy is still in its infancy and the complexities of pathogenic signaling need to be resolved to initially understand these proinflammatory subpopulations, maintaining the protective - regulatory cells [95] and even how we can target the neurodegenerative changes. BAFF binding to $\mathrm{NgR}$ may indeed be a plausible target for chronic active MS treatment. Since the BAFF and APRIL system can interact with components of $\mathrm{T}$ and $\mathrm{B}$ cell pathways of chronic inflammatory disorders targeting a novel signaling cascade that may potentiate autoimmunity warrants further investigation. Considering that $\mathrm{NgR} 1+$ and $\mathrm{NgR} 3+\mathrm{B}$ cells maybe involved in humoral mediated neuroinflammatory events, it is clear that therapeutic treatments against the immune responses that are myelin orientated may be developed.

\section{Acknowledgements ORCID}

- Paschalis Theotokis: 0000-0001-8607-6695;

- Steven Petratos: 0000-0003-1211-4577.

\section{Competing Interests}

The authors have declared that no competing interest exists.

\section{References}

1. Li R, Patterson KR, Bar-Or A. Reassessing B cell contributions in multiple sclerosis. Nat Immunol. 2018; 19: 696-707.

2. Filippi M, Bar-Or A, Piehl F, Preziosa P, Solari A, Vukusic S, et al. Multiple sclerosis. Nat Rev Dis Primers. 2018; 4: 43.

3. Klineova S, Lublin FD. Clinical Course of Multiple Sclerosis. Cold Spring Harb Perspect Med. 2018; 8.

4. Comi G, Bar-Or A, Lassmann H, Uccelli A, Hartung HP, Montalban X, et al. Role of B Cells in Multiple Sclerosis and Related Disorders. Ann Neurol. 2021; 89: 13-23

5. Hauser SL, Waubant E, Arnold DL, Vollmer T, Antel J, Fox RJ, et al. B-cell depletion with rituximab in relapsing-remitting multiple sclerosis. N Engl J Med. 2008; 358: 676-88.

6. Kappos L, Li D, Calabresi PA, O'Connor P, Bar-Or A, Barkhof F, et al. Ocrelizumab in relapsing-remitting multiple sclerosis: a phase 2, randomised, placebo-controlled, multicentre trial. Lancet. 2011; 378: 1779-87.

7. Magliozzi R, Columba-Cabezas S, Serafini B, Aloisi F. Intracerebral expression of CXCL13 and BAFF is accompanied by formation of lymphoid follicle-like structures in the meninges of mice with relapsing experimental autoimmune encephalomyelitis. J Neuroimmunol. 2004; 148: 11-23.

8. Thangarajh M, Gomes A, Masterman T, Hillert J, Hjelmstrom P. Expression of B-cell-activating factor of the TNF family (BAFF) and its receptors in multiple sclerosis. J Neuroimmunol. 2004; 152: 183-90.

9. Hoglund RA, Maghazachi AA. Multiple sclerosis and the role of immune cells. World J Exp Med. 2014; 4: 27-37.

10. McFarland HF, Martin R. Multiple sclerosis: a complicated picture of autoimmunity. Nat Immunol. 2007; 8: 913-9.

11. Steinman L. A brief history of $\mathrm{T}(\mathrm{H}) 17$, the first major revision in the $\mathrm{T}(\mathrm{H}) 1 / \mathrm{T}(\mathrm{H}) 2$ hypothesis of $\mathrm{T}$ cell-mediated tissue damage. Nat Med. 2007; 13: 139-45.

12. Viglietta V, Baecher-Allan C, Weiner HL, Hafler DA. Loss of functional suppression by CD4+CD25+ regulatory T cells in patients with multiple sclerosis. J Exp Med. 2004; 199: 971-9.

13. Vasileiadis GK, Dardiotis E, Mavropoulos A, Tsouris Z, Tsimourtou V, Bogdanos DP, et al. Regulatory B and T lymphocytes in multiple sclerosis: friends or foes? Auto Immun Highlights. 2018; 9: 9.
14. Puthenparampil M, Federle L, Miante S, Zito A, Toffanin E, Ruggero S, et al. BAFF Index and CXCL13 levels in the cerebrospinal fluid associate respectively with intrathecal IgG synthesis and cortical atrophy in multiple sclerosis at clinical onset. J Neuroinflammation. 2017; 14: 11.

15. Magliozzi R, Howell O, Vora A, Serafini B, Nicholas R, Puopolo M, et al. Meningeal B-cell follicles in secondary progressive multiple sclerosis associate with early onset of disease and severe cortical pathology. Brain. 2007; 130: 1089-104.

16. Haugen M, Frederiksen JL, Degn M. B cell follicle-like structures in multiple sclerosis-with focus on the role of B cell activating factor. J Neuroimmunol. 2014; 273: 1-7.

17. Howell OW, Reeves CA, Nicholas R, Carassiti D, Radotra B, Gentleman $\mathrm{SM}$, et al. Meningeal inflammation is widespread and linked to cortical pathology in multiple sclerosis. Brain. 2011; 134: 2755-71.

18. Mackay F, Schneider P. Cracking the BAFF code. Nat Rev Immunol. 2009; 9: 491-502.

19. Sakai J, Akkoyunlu M. The Role of BAFF System Molecules in Host Response to Pathogens. Clin Microbiol Rev. 2017; 30: 991-1014.

20. Smulski CR, Eibel H. BAFF and BAFF-Receptor in B Cell Selection and Survival. Front Immunol. 2018; 9: 2285.

21. Petratos S, Ozturk E, Azari MF, Kenny R, Lee JY, Magee KA, et al. Limiting multiple sclerosis related axonopathy by blocking Nogo receptor and CRMP-2 phosphorylation. Brain. 2012; 135: 1794-818.

22. Constantinescu CS, Farooqi N, O'Brien K, Gran B. Experimental autoimmune encephalomyelitis (EAE) as a model for multiple sclerosis (MS). Br J Pharmacol. 2011; 164: 1079-106.

23. Ortega SB, Kashi VP, Tyler AF, Cunnusamy K, Mendoza JP, Karandikar NJ. The disease-ameliorating function of autoregulatory CD8 T cells is mediated by targeting of encephalitogenic CD4 T cells in experimental autoimmune encephalomyelitis. J Immunol. 2013; 191: 117-26.

24. Machado-Santos J, Saji E, Troscher AR, Paunovic M, Liblau R, Gabriely $\mathrm{G}$, et al. The compartmentalized inflammatory response in the multiple sclerosis brain is composed of tissue-resident CD8+ T lymphocytes and B cells. Brain. 2018; 141: 2066-82.

25. Sabatino JJ, Jr., Wilson MR, Calabresi PA, Hauser SL, Schneck JP, Zamvil SS. Anti-CD20 therapy depletes activated myelin-specific CD8(+) T cells in multiple sclerosis. Proc Natl Acad Sci U S A. 2019; 116: 25800-7.

26. Pachner AR, Li L, Lagunoff D. Plasma cells in the central nervous system in the Theiler's virus model of multiple sclerosis. J Neuroimmunol. 2011; 232: 35-40.

27. Friese MA, Jakobsen KB, Friis L, Etzensperger R, Craner MJ, McMahon $\mathrm{RM}$, et al. Opposing effects of HLA class I molecules in tuning autoreactive CD8+ T cells in multiple sclerosis. Nat Med. 2008; 14: 1227-35.

28. Dang AK, Tesfagiorgis Y, Jain RW, Craig HC, Kerfoot SM. Meningeal Infiltration of the Spinal Cord by Non-Classically Activated B Cells is Associated with Chronic Disease Course in a Spontaneous B Cell-Dependent Model of CNS Autoimmune Disease. Front Immunol. 2015; 6: 470.

29. Magliozzi R, Marastoni D, Calabrese M. The BAFF / APRIL system as therapeutic target in multiple sclerosis. Expert Opin Ther Targets. 2020; 24: $1135-45$

30. Krumbholz M, Theil D, Derfuss T, Rosenwald A, Schrader F, Monoranu $\mathrm{CM}$, et al. BAFF is produced by astrocytes and up-regulated in multiple sclerosis lesions and primary central nervous system lymphoma. J Exp Med. 2005; 201: 195-200.

31. Franciotta D, Di Stefano AL, Jarius S, Zardini E, Tavazzi E, Ballerini C, et al. Cerebrospinal BAFF and Epstein-Barr virus-specific oligoclonal bands in multiple sclerosis and other inflammatory demyelinating neurological diseases. J Neuroimmunol. 2011; 230: 160-3.

32. Franciotta D, Salvetti M, Lolli F, Serafini B, Aloisi F. B cells and multiple sclerosis. Lancet Neurol. 2008; 7: 852-8.

33. Steri M, Orru V, Idda ML, Pitzalis M, Pala M, Zara I, et al. Overexpression of the Cytokine BAFF and Autoimmunity Risk. N Engl J Med. 2017; 376: 1615-26.

34. Badr G, Borhis G, Lefevre EA, Chaoul N, Deshayes F, Dessirier V, et al. BAFF enhances chemotaxis of primary human B cells: a particular synergy between BAFF and CXCL13 on memory B cells. Blood. 2008; 111: 2744-54.

35. Kowarik MC, Cepok S, Sellner J, Grummel V, Weber MS, Korn T, et al. CXCL13 is the major determinant for B cell recruitment to the CSF during neuroinflammation. J Neuroinflammation. 2012; 9: 93.

36. Ragheb S, Li Y, Simon K, VanHaerents S, Galimberti D, De Riz M, et al. Multiple sclerosis: BAFF and CXCL13 in cerebrospinal fluid. Mult Scler. 2011; 17: 819-29.

37. Swee LK, Tardivel A, Schneider P, Rolink A. Rescue of the mature B cell compartment in BAFF-deficient mice by treatment with recombinant Fc-BAFF. Immunol Lett. 2010; 131: 40-8. 
38. Hengeveld PJ, Kersten MJ. B-cell activating factor in the pathophysiology of multiple myeloma: a target for therapy? Blood Cancer J. 2015; 5: e282.

39. Laabi Y, Egle A, Strasser A. TNF cytokine family: more BAFF-ling complexities. Curr Biol. 2001; 11: R1013-6.

40. Mackay F, Browning JL. BAFF: a fundamental survival factor for B cells. Nat Rev Immunol. 2002; 2: 465-75.

41. Schneider P, MacKay F, Steiner V, Hofmann K, Bodmer JL, Holler N, et al. BAFF, a novel ligand of the tumor necrosis factor family, stimulates B cell growth. J Exp Med. 1999; 189: 1747-56.

42. Bossen C, Schneider P. BAFF, APRIL and their receptors: structure, function and signaling. Semin Immunol. 2006; 18: 263-75.

43. Kalled SL, Ambrose C, Hsu YM. The biochemistry and biology of BAFF, APRIL and their receptors. Curr Dir Autoimmun. 2005; 8: 206-42.

44. Jackson SW, Davidson A. BAFF inhibition in SLE-Is tolerance restored? Immunol Rev. 2019; 292: 102-19.

45. Vigolo M, Chambers MG, Willen L, Chevalley D, Maskos K, Lammens $\mathrm{A}$, et al. A loop region of BAFF controls $\mathrm{B}$ cell survival and regulates recognition by different inhibitors. Nat Commun. 2018; 9: 1199.

46. Vincent FB, Morand EF, Schneider P, Mackay F. The BAFF/APRIL system in SLE pathogenesis. Nat Rev Rheumatol. 2014; 10: 365-73.

47. Vincent FB, Morand EF, Mackay F. BAFF and innate immunity: new therapeutic targets for systemic lupus erythematosus. Immunol Cell Biol. 2012; 90: 293-303.

48. Shabgah AG, Shariati-Sarabi Z, Tavakkol-Afshari J, Mohammadi M. The role of BAFF and APRIL in rheumatoid arthritis. J Cell Physiol. 2019; 234: 17050-63

49. Craxton A, Magaletti D, Ryan EJ, Clark EA. Macrophage- and dendritic cell--dependent regulation of human B-cell proliferation requires the TNF family ligand BAFF. Blood. 2003; 101: 4464-71.

50. Ittah M, Miceli-Richard C, Eric Gottenberg J, Lavie F, Lazure T, Ba N, et al. B cell-activating factor of the tumor necrosis factor family (BAFF) is expressed under stimulation by interferon in salivary gland epithelial cells in primary Sjogren's syndrome. Arthritis Res Ther. 2006; 8: R51.

51. Kato A, Truong-Tran AQ, Scott AL, Matsumoto K, Schleimer RP. Airway epithelial cells produce B cell-activating factor of TNF family by an IFN-beta-dependent mechanism. J Immunol. 2006; 177: 7164-72.

52. Mackay F, Silveira PA, Brink R. B cells and the BAFF/APRIL axis: fast-forward on autoimmunity and signaling. Curr Opin Immunol. 2007; 19: 327-36.

53. Schaumann $\mathrm{DH}$, Tuischer $\mathrm{J}$, Ebell $\mathrm{W}$, Manz RA, Lauster $\mathrm{R}$. VCAM-1-positive stromal cells from human bone marrow producing cytokines for B lineage progenitors and for plasma cells: SDF-1, flt3L, and BAFF. Mol Immunol. 2007; 44: 1606-12.

54. Ohata J, Zvaifler NJ, Nishio M, Boyle DL, Kalled SL, Carson DA, et al. Fibroblast-like synoviocytes of mesenchymal origin express functional B cell-activating factor of the TNF family in response to proinflammatory cytokines. J Immunol. 2005; 174: 864-70.

55. Mackay F, Schneider P, Rennert P, Browning J. BAFF AND APRIL: a tutorial on B cell survival. Annu Rev Immunol. 2003; 21: 231-64.

56. Schiemann B, Gommerman JL, Vora K, Cachero TG, Shulga-Morskaya S, Dobles $\mathrm{M}$, et al. An essential role for BAFF in the normal development of B cells through a BCMA-independent pathway. Science. 2001; 293: 2111-4.

57. Khan WN. B cell receptor and BAFF receptor signaling regulation of B cell homeostasis. J Immunol. 2009; 183: 3561-7.

58. Yan M, Wang H, Chan B, Roose-Girma M, Erickson S, Baker T, et al. Activation and accumulation of B cells in TACI-deficient mice. Nat Immunol. 2001; 2: 638-43.

59. Dhaya Seshasayee PV, Minhong Yan, Vishva M Dixit, Daniel Tumas, Iqbal S Grewal. Loss of TACI causes fatal lymphoproliferation and autoimmunity, establishing TACI as an inhibitory BLyS receptor. Immunity. 2003 18: 279-88

60. Avery DT, Kalled SL, Ellyard JI, Ambrose C, Bixler SA, Thien M, et al. BAFF selectively enhances the survival of plasmablasts generated from human memory B cells. J Clin Invest. 2003; 112: 286-97.

61. O'Connor BP, Raman VS, Erickson LD, Cook WJ, Weaver LK, Ahonen C, et al. BCMA is essential for the survival of long-lived bone marrow plasma cells. J Exp Med. 2004; 199: 91-8.

62. Mackay F, Figgett WA, Saulep D, Lepage M, Hibbs ML. B-cell stage and context-dependent requirements for survival signals from BAFF and the B-cell receptor. Immunol Rev. 2010; 237: 205-25.

63. Zhang Q, Lenardo MJ, Baltimore D. 30 Years of NF-kappaB: A Blossoming of Relevance to Human Pathobiology. Cell. 2017; 168: 37-57.

64. Claudio E, Brown K, Park S, Wang H, Siebenlist U. BAFF-induced NEMO-independent processing of NF-kappa B2 in maturing B cells. Nat Immunol. 2002; 3: 958-65.

65. Xiao G, Harhaj EW, Sun SC. NF-kappaB-inducing kinase regulates the processing of NF-kappaB2 p100. Mol Cell. 2001; 7: 401-9.
66. Jellusova J, Miletic AV, Cato MH, Lin WW, Hu Y, Bishop GA, et al. Context-specific BAFF-R signaling by the NF-kappaB and PI3K pathways. Cell Rep. 2013; 5: 1022-35.

67. Schweighoffer E, Vanes L, Nys J, Cantrell D, McCleary S, Smithers N, et al. The BAFF receptor transduces survival signals by co-opting the B cell receptor signaling pathway. Immunity. 2013; 38: 475-88.

68. Woodland RT, Fox CJ, Schmidt MR, Hammerman PS, Opferman JT, Korsmeyer SJ, et al. Multiple signaling pathways promote B lymphocyte stimulator dependent B-cell growth and survival. Blood. 2008; 111: 750-60.

69. Castigli E, Alt FW, Davidson L, Bottaro A, Mizoguchi E, Bhan AK, et al. CD40-deficient mice generated by recombination-activating gene-2-deficient blastocyst complementation. Proc Natl Acad Sci U S A. 1994; 91: 12135-9.

70. Litinskiy MB, Nardelli B, Hilbert DM, He B, Schaffer A, Casali P, et al. DCs induce CD40-independent immunoglobulin class switching through BLyS and APRIL. Nat Immunol. 2002; 3: 822-9.

71. Diaz-de-Durana Y, Mantchev GT, Bram RJ, Franco A. TACI-BLyS signaling via B-cell-dendritic cell cooperation is required for naive CD8+ T-cell priming in vivo. Blood. 2006; 107: 594-601.

72. Zheng Y, Gallucci S, Gaughan JP, Gross JA, Monestier M. A role for B cell-activating factor of the TNF family in chemically induced autoimmunity. J Immunol. 2005; 175: 6163-8.

73. Gross JA, Johnston J, Mudri S, Enselman R, Dillon SR, Madden K, et al. TACI and BCMA are receptors for a TNF homologue implicated in B-cell autoimmune disease. Nature. 2000; 404: 995-9.

74. Zhang M, Ko KH, Lam QL, Lo CK, Srivastava G, Zheng B, et al. Expression and function of TNF family member B cell-activating factor in the development of autoimmune arthritis. Int Immunol. 2005; 17: 1081-92.

75. Zhang L, Zheng S, Wu H, Wu Y, Liu S, Fan M, et al. Identification of BLyS (B lymphocyte stimulator), a non-myelin-associated protein, as a functional ligand for Nogo-66 receptor. J Neurosci. 2009; 29: 6348-52.

76. Fournier AE, GrandPre T, Strittmatter SM. Identification of a receptor mediating Nogo-66 inhibition of axonal regeneration. Nature. 2001; 409: 341-6.

77. Bakhuraysah MM, Theotokis P, Lee JY, Alrehaili AA, Aui PM, Figgett WA, et al. B-cells expressing $\mathrm{NgR} 1$ and $\mathrm{NgR} 3$ are localized to EAE-induced inflammatory infiltrates and are stimulated by BAFF. Sci Rep. 2021; 11: 2890

78. Theotokis P, Grigoriadis N. p75NTR and TROY: Uncharted Roles of Nogo Receptor Complex in Experimental Autoimmune Encephalomyelitis. Mol Neurobiol. 2018; 55: 6329-36.

79. Theotokis P, Touloumi O, Lagoudaki R, Nousiopoulou E, Kesidou E, Siafis S, et al. Nogo receptor complex expression dynamics in the inflammatory foci of central nervous system experimental autoimmune demyelination. J Neuroinflammation. 2016; 13: 265.

80. Petratos S, Azari MF, Ozturk E, Papadopoulos R, Bernard CC. Novel therapeutic targets for axonal degeneration in multiple sclerosis. J Neuropathol Exp Neurol. 2010; 69: 323-34.

81. Saha N, Kolev M, Nikolov DB. Structural features of the Nogo receptor signaling complexes at the neuron/myelin interface. Neurosci Res. 2014; 87: $1-7$

82. McDonald CL, Bandtlow C, Reindl M. Targeting the Nogo receptor complex in diseases of the central nervous system. Curr Med Chem. 2011; 18: 234-44.

83. Grandpre T, Strittmatter SM. Nogo: a molecular determinant of axonal growth and regeneration. Neuroscientist. 2001; 7: 377-86.

84. Theotokis P, Lourbopoulos A, Touloumi O, Lagoudaki R, Kofidou E, Nousiopoulou E, et al. Time course and spatial profile of Nogo-A expression in experimental autoimmune encephalomyelitis in C57BL/6 mice. J Neuropathol Exp Neurol. 2012; 71: 907-20.

85. Petratos S, Theotokis P, Kim MJ, Azari MF, Lee JY. That's a Wrap! Molecular Drivers Governing Neuronal Nogo Receptor-Dependent Myelin Plasticity and Integrity. Front Cell Neurosci. 2020; 14: 227.

86. Park JB, Yiu G, Kaneko S, Wang J, Chang J, He XL, et al. A TNF receptor family member, TROY, is a coreceptor with Nogo receptor in mediating the inhibitory activity of myelin inhibitors. Neuron. 2005; 45: 345-51.

87. Kim MJ, Kang JH, Theotokis P, Grigoriadis N, Petratos S. Can We Design a Nogo Receptor-Dependent Cellular Therapy to Target MS? Cells. 2018; 8.

88. Freedman MS, Selchen D, Prat A, Giacomini PS. Managing Multiple Sclerosis: Treatment Initiation, Modification, and Sequencing. Can J Neurol Sci. 2018; 45: 489-503.

89. Gholamzad M, Ebtekar M, Ardestani MS, Azimi M, Mahmodi Z, Mousavi MJ, et al. A comprehensive review on the treatment approaches of multiple sclerosis: currently and in the future. Inflamm Res. 2019; 68: 25-38. 
90. Pilz G, Wipfler P, Ladurner G, Kraus J. Modern multiple sclerosis treatment - what is approved, what is on the horizon. Drug Discov Today. 2008; 13: 1013-25.

91. Tintore M, Vidal-Jordana A, Sastre-Garriga J. Treatment of multiple sclerosis - success from bench to bedside. Nat Rev Neurol. 2019; 15: 53-8.

92. Li H, Hu F, Zhang Y, Li K. Comparative efficacy and acceptability of disease-modifying therapies in patients with relapsing-remitting multiple sclerosis: a systematic review and network meta-analysis. J Neurol. 2020; 267: 3489-98.

93. Reich DS, Lucchinetti CF, Calabresi PA. Multiple Sclerosis. N Engl J Med. 2018; 378: 169-80.

94. Hauser SL, Cree BAC. Treatment of Multiple Sclerosis: A Review. Am J Med. 2020; 133: 1380-90 e2.

95. Rahmanzadeh R, Weber MS, Bruck W, Navardi S, Sahraian MA. B cells in multiple sclerosis therapy-A comprehensive review. Acta Neurol Scand. 2018; 137: 544-56.

96. Dobson R, Giovannoni G. Multiple sclerosis - a review. Eur J Neurol. 2019; 26: 27-40.

97. Huntington ND, Tomioka R, Clavarino C, Chow AM, Linares D, Mana P, et al. A BAFF antagonist suppresses experimental autoimmune encephalomyelitis by targeting cell-mediated and humoral immune responses. Int Immunol. 2006; 18: 1473-85.

98. Krumbholz M, Derfuss T, Hohlfeld R, Meinl E. B cells and antibodies in multiple sclerosis pathogenesis and therapy. Nat Rev Neurol. 2012; 8: 613-23.

99. Kaegi C, Steiner UC, Wuest B, Crowley C, Boyman O. Systematic Review of Safety and Efficacy of Atacicept in Treating Immune-Mediated Disorders. Front Immunol. 2020; 11: 433.

100. Kappos L, Hartung HP, Freedman MS, Boyko A, Radu EW, Mikol DD, et al. Atacicept in multiple sclerosis (ATAMS): a randomised, placebo-controlled, double-blind, phase 2 trial. Lancet Neurol. 2014; 13: 353-63.

101. Stohl W, Hilbert DM. The discovery and development of belimumab: the anti-BLyS-lupus connection. Nat Biotechnol. 2012; 30: 69-77.

102. Fitzner D, Simons M. Chronic progressive multiple sclerosis pathogenesis of neurodegeneration and therapeutic strategies. Curr Neuropharmacol. 2010; 8: 305-15. 